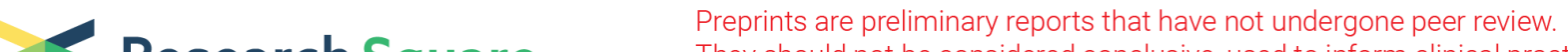 Research Square They should not be considered conclusive, used to inform clinical practice, or referenced by the media as validated information.
}

\section{Slope Analysis for the Prediction of Fluid Responsiveness by a Stepwise PEEP Elevation Recruitment Maneuver in Mechanically Ventilated Patients}

Sylvain VALLIER ( $\square$ vallier.sylvain@gmail.com )

Elsan Alpes-Belledonne Clinic

Jean-Baptiste BOUCHET

Saint-Etienne University Hospital, Jean-Monnet University

Olivier DESEBBE

Ramsay Sante Sauvegarde Clinic

Camille FRANCOU

Saint-Etienne University Hospital, Jean-Monnet University

Darren RAPHAEL

University of California, Irvine

Bernard TARDY

Centre d'Investigation Clinique - CIC 1408, Saint-Etienne University Hospital, Jean-Monnet University

Laurent GERGELE

Ramsay Sante HPL Clinic

Jérôme MOREL

Saint-Etienne University Hospital, Jean-Monnet University

\section{Research Article}

Keywords: Lung recruitment maneuver, fluid responsiveness, central venous pressure, pulse pressure, hemodynamics, mechanical ventilation

Posted Date: October 1st, 2021

DOI: https://doi.org/10.21203/rs.3.rs-125412/v2

License: (c) (1) This work is licensed under a Creative Commons Attribution 4.0 International License. Read Full License

Version of Record: A version of this preprint was published at BMC Anesthesiology on January 3rd, 2022. See the published version at https://doi.org/10.1186/s12871-021-01544-x. 


\section{Abstract}

\section{Objective:}

Assessment of fluid responsiveness is problematic in intensive care unit patients. Lung recruitment maneuvers (LRM) can be used as a functional test to predict fluid responsiveness. We propose a new test to predict fluid responsiveness in mechanically ventilated patients by analyzing the variations in central venous pressure (CVP) and systemic arterial parameters during a prolonged sigh breath LRM without the use of a cardiac output measuring device.

\section{Design:}

Prospective observational cohort study.

\section{Setting:}

Intensive Care Unit, Saint-Etienne University Central Hospital.

\section{Patients:}

Patients under mechanical ventilation, equipped with invasive arterial blood pressure, CVP, pulse contour analysis $\left(\mathrm{PICCO}^{\mathrm{TM}}\right)$, requiring volume expansion, with no right ventricular dysfunction.

\section{Interventions:}

None.

\section{Measurements and Main Results:}

CVP, systemic arterial parameters and stroke volume (SV) were recorded during prolonged LRM followed by a $500 \mathrm{~mL}$ fluid expansion to asses fluid responsiveness. 25 patients were screened and 18 patients analyzed. 9 patients were responders to volume expansion and 9 were not. Evaluation of hemodynamic parameters suggested the use of a linear regression model. Slopes for systolic arterial pressure, pulse pressure (PP), CVP and SV were all significantly different between responders and non-responders during the pressure increase phase of LRM (STEP-UP) $(p=0.022, p=0.014, p=0.006$ and $p=0.038$, respectively). PP and CVP slopes during STEP-UP were strongly predictive of fluid responsiveness with an AUC of 0.926 $(95 \% \mathrm{Cl}, 0.78$ to 1.00$)$, sensitivity $=100 \%$, specificity $=89 \%$ and an $\mathrm{AUC}=0.901$ (95\% Cl, 0.76 to 1.00$)$, sensibility $=78 \%$, specificity $=100 \%$, respectively. Combining sensitivity of PP and specificity of CVP, prediction of fluid responsiveness can be achieved with $100 \%$ sensitivity and $100 \%$ specificity (AUC $=0.96$; $95 \% \mathrm{Cl}, 0.90$ to 1.00$)$. One patient showed inconclusive values using the grey zone approach (5.5\%).

\section{Conclusions:}

In patients under mechanical ventilation with no right heart dysfunction, the association of PP and CVP slope analysis during a prolonged sigh breath LRM seems to offer a very promising method for prediction 
of fluid responsiveness without the use and associated cost of a cardiac output measurement device.

\section{Introduction}

Hemodynamic and fluid optimization during the perioperative period has been shown to reduce postoperative morbidity. (1) Unfortunately, the assessment of preload and determination of whether the patient will be fluid responsive has proved challenging. Static preload indices such as central venous pressure are not sufficient to assess fluid responsiveness (2), whereas dynamic preload indices such as pulse pressure variation (PPV) and stroke volume (SV) variation have been used successfully. (3) However, such indices suffer from several limitations and should be used only under specific conditions. (4) Alternative dynamic methods of assessment such as respiratory systolic variation test (RSVT) (5) and lung recruitment maneuvers (LRM) have been developed. (6) LRM can be used to reopen or prevent atelectasis during mechanical ventilation in order to decrease respiratory complications. (7) LRM induce a transient increase in intra-thoracic pressure and a decrease in venous return, leading to a decrease in left ventricular end-diastolic area and stroke volume. $(8,9)$ Several studies have shown that the PEEP-induced decrease in stroke volume is related to pre-existing preload responsiveness. $(10,11)$ A few studies have also shown that LRM can be used as a functional test to predict fluid responsiveness. $(12,13,14)$ However, monitoring stroke volume during LRM to assess fluid responsiveness is costly, and cardiac output devices may not be reliable. (15) In this context, central venous pressure (CVP) or systemic arterial monitoring represents a cost effective and readily available alternative for predicting fluid responsiveness during major surgery.

LRM can be performed using a prolonged sigh breath or stepwise increase in PEEP and airway inspiratory pressure with a constant drive pressure. $(16,17)$ These maneuvers have been described for 2 to 4 -minute periods. Prolonged LRM leads to a smaller increase in transpulmonary pressure for a longer period of time and improves lung aeration as effectively as sustained inflation, with less risk of hemodynamic compromise or hyperinflation. A recent study has specifically evaluated the hemodynamic response in this context. (18)

The aims of the current study were (1) to predict fluid responsiveness using changes in hemodynamic measurements during a stepwise increase in PEEP LRM in mechanically ventilated patients, (2) to identify the best criteria for predicting fluid responsiveness among changes in systolic arterial pressure (SAP), mean arterial pressure (MAP), diastolic arterial pressure (DAP), pulse pressure (PP) and central venous pressure (CVP), and (3) to compare the ability of these criteria with pulse pressure variation (PPV) to predict fluid responsiveness.

\section{Material And Methods}

We conducted a prospective study in the 23-bed intensive care unit (ICU) at Saint-Etienne University Medical Center, France, between December 2019 and December 2020. The study protocol was approved by the hospital's ethics committee (Ethics Committee, Department of Anesthesiology, Saint-Etienne University Central Hospital, institutional Review Board IORG0007394, Protocol number IRBN902018/CHUSTE). All methods were performed in accordance with the relevant guidelines and regulations. Written informed 
consent was obtained for all study patients or relatives if indicated. Oral consent was obtained and reported in the medical record. Inclusion criteria were as follows: invasive arterial blood pressure and pulse contour analysis (PICCO system, Pulsion Medical Systems SE, Feldkirchen, Germany) for cardiac output measurement, central venous pressure monitoring, use of protective mechanical ventilation, age greater than 18 years and indication for volume expansion. A transthoracic echocardiography was performed on all patients prior to inclusion. Non-inclusion criteria were right ventricular dysfunction, significant valvulopathy, ejection fraction less than $50 \%$, arrhythmia or presence of spontaneous breathing cycles. The inclusion time was at the start of the LRM. Exclusion criteria were: LRM not completed, absence of fluid expansion performed after LRM and patient decline for enrollment after reawakening.

\section{Sedation \& monitoring}

Each patient was monitored with pulse oximetry and a 5-lead EKG. Central venous pressure was measured continuously for all patients. All patients were equipped with a Transcardiopulmonary ThermodilutionCalibrated Arterial Waveform Analysis (PICCO system) inserted into a femoral artery. Pressure transducers were placed at the level of the mid-axillary line throughout the study protocol. All patients were intubated and ventilated using a volume-controlled mode. Sedation was maintained with propofol and/or midazolam in combination with either sufentanil or remifentanil. Neuromuscular blockade was not systematically used. The tidal volume (TV) was set by the clinician to the ideal body weight to obtain [6-8] $\mathrm{mL} / \mathrm{kg}$ and the ventilatory rate was set in order to maintain arterial CO2 tension between 35 and $45 \mathrm{mmHg}$.

\section{Lung recruitment maneuver}

LRM were performed using a stepwise increase in PEEP and airway inspiratory pressure with the same drive pressure $(15 \mathrm{cmH} 20)$, as described in the literature. $(15,16,18)$ The LRM consisted of a $5 \mathrm{cmH}_{2} \mathrm{O}$ PEEP and inspiratory pressure increase every 30 seconds. The baseline was $5 \mathrm{cmH}_{2} \mathrm{O}$ PEEP and $20 \mathrm{cmH}_{2} \mathrm{O}$ inspiratory pressure for all patients. The maximum pressure level reached was $30 \mathrm{cmH}_{2} \mathrm{O}$ PEEP and $45 \mathrm{cmH} 2 \mathrm{O}$ inspiratory pressure. PEEP de-escalation was performed following the same pattern (Figure 1). After LRM, ventilatory settings were set back to initial patient settings. We defined the increasing levels of pressure as "STEP-UP" and the decreasing levels of pressure as "STEP-DOWN". LRM were stopped if severe arterial hypotension (systolic arterial pressure less than $70 \mathrm{mmHg}$ ) or severe hypoxemia $(\mathrm{SpO} 2<80 \%$ ) was observed. (17) All patients considered for analysis received a fluid expansion after LRM. Fluid challenge was standardized and consisted of Plasmalyte infusion of $500 \mathrm{ml}$ over 10 minutes.

\section{Data collection}

We recorded demographic data including weight, age, gender, Simplified Acute Physiology Score (SAPS II), medical history, criteria of admission to ICU and comorbidities. Respiratory parameters (tidal volume, respiratory rate, insufflation pressure [peak], plateau pressure, level of PEEP), hemodynamic parameters (SAP, DAP, MAP), pulse pressure (PP=SAP-DAP), CVP, heart rate, PPV, and PICCO data (stroke volume and cardiac output (CO)) were also recorded. Baseline PPV was displayed on Phillips monitors.

Pharmacological data (vasoactive infusions) and biological data (lactate) were recorded. 


\section{Study protocol}

When a patient met the inclusion criteria, the investigating physicians collected a set of demographic, ventilatory and hemodynamic data. A transcardiopulmonary thermodilution was performed. Three injections of $20 \mathrm{~mL}$ cold fluid bolus were used for SV and CO calculations at baseline (mean of the three bolus). The LRM was then performed following the STEP-PEEP pattern as described above. A video of the hemodynamic monitoring was recorded during the LRM, with the clinician announcing the time and pressure level for each step. Hemodynamic values were later documented by pausing on the video during the two last seconds of each LRM step as announced by the physician. Next, a fluid expansion of PlasmaLyte $500 \mathrm{~mL}$ over 10 minutes was performed. A second transcardiopulmonary thermodilution was done between 2 to 4 minutes after fluid expansion, using the same method. Responders (R) and NonResponders (NR) were defined with regard to the change in SV (expressed as percentage) after fluid expansion. A fluid responder was defined as a $15 \%$ increase in SV after fluid expansion. (20)

\section{Statistics}

A sample size of 18 patients ( 9 responders and 9 non-responders) was calculated to be sufficient to demonstrate that CVP and PP variations can predict fluid responsiveness with an area under curve (AUC) of 0.85 , a power of $80 \%$ (beta risk $=0.2$ ) and an alpha risk of 0.05 . Data are expressed as mean (SD) or median (25th to 75th ). We used the Student T-test for continuous variables. Slope values were obtained using a linear regression calculation are expressed in degrees for the angle (a) between the horizontal axis and the linear regression curve calculated between the drop of pressure $(\mathrm{mmHg})$ and the PEEP level (cmH2O). The threshold for statistical significance was set to $\mathrm{P}<0.05$. A receiver-operating characteristic (ROC) curve was drawn for aSAP, aMAP, aDAP, aPP and aCVP for STEP-UP and STEP-DOWN during LRM, respectively. We selected the threshold that gave the highest Youden index. We defined inconclusive responses (grey zone) for values within the $\mathrm{Cl} 95 \%$ of the obtained threshold value according to Cannesson et al. (21) The method described by DeLong et al was used to compare the areas under the ROC curve associated with the variables. (22) Statistical analyses were performed with XLSTAT software (version 2019.3.2).

\section{Results}

\section{Patient characteristics}

A total of 25 nonconsecutive patients were screened. Five patients were not included due to right ventricular dysfunction (1 patient), ejection fraction less than $50 \%$ ( 1 patient) and presence of arrythmia (3 patients). Two patients were excluded due to absence of fluid expansion performed after LRM. A total of 18 patients were analyzed (Figure 2). Nine patients (50\%) were responders to volume expansion and nine were not. Patient main characteristics, hemodynamic, respiratory, pharmacological and biological variables in both Responders and Non-Responders are shown in Table 1. The PPV value was not calculated for 8 patients.

The baseline norepinephrine concentration was higher in the Responders group (R) than in the NonResponders (NR) group $(0.78 \mathrm{ug} / \mathrm{kg} / \mathrm{min}$ vs $0.24 \mathrm{ug} / \mathrm{kg} / \mathrm{min}$, respectively; $\mathrm{p}=0.031)$. Baseline PPV was 
higher in the $R$ than in the NR group ( $14 \%$ vs $6 \%$, respectively; $p=0.034$ ). $R$ and NR did not differ for baseline values of SV, CO, MAP, CVP or lactate level. Static pulmonary compliance was not statistically different between $\mathrm{R}$ and $\mathrm{NR}$. 
Table 1

Patient demographic data, hemodynamic, respiratory, pharmacological and biological variables at baseline in Responders $(n=9)$ and Non-Responders ( $n=9$ patients)

\begin{tabular}{|c|c|c|c|c|}
\hline & $\begin{array}{l}\text { Overall } \\
\text { population }\end{array}$ & Responders & $\begin{array}{l}\text { Non- } \\
\text { Responders }\end{array}$ & $\begin{array}{l}\mathrm{p} \\
\text { value }\end{array}$ \\
\hline & $\mathrm{N}=18$ & $\mathrm{~N}=9$ & $\mathrm{~N}=9$ & \\
\hline Age (mean SD), yr & $60(15)$ & $66(7)$ & $57(18)$ & \\
\hline Gender (M/F) & $10 / 8$ & $5 / 4$ & $5 / 4$ & \\
\hline BMI (mean SD), kg.m-2 & $29(9)$ & $29(6)$ & $29(12)$ & \\
\hline Ideal body weight (mean SD), kg & $66(14)$ & $63(11)$ & $66(17)$ & \\
\hline SAPS 2 (mean SD) & $61(22)$ & $69(22)$ & $57(21)$ & \\
\hline \multicolumn{5}{|l|}{ ICU admission criteria } \\
\hline Septic shock & 4 & 3 & 1 & \\
\hline Cardiac failure & 7 & 3 & 4 & \\
\hline Respiratory failure & 4 & 2 & 2 & \\
\hline Hemorrhagic shock & 1 & 1 & 0 & \\
\hline Polytrauma & 1 & 0 & 0 & \\
\hline Cranio-cerebral trauma & 1 & 0 & 1 & \\
\hline \multicolumn{5}{|l|}{ Comorbidities } \\
\hline Arterial hypertension & 8 & 6 & 2 & \\
\hline Diabetes & 4 & 2 & 2 & \\
\hline Coronary artery disease & 1 & 1 & 0 & \\
\hline \multicolumn{5}{|l|}{ Hemodynamic parameters } \\
\hline Mean arterial pressure (mean SD), mmHg & $73(9)$ & $70(10)$ & $76(7)$ & 0.110 \\
\hline Heart Rate (mean SD), HR/min & $90(18)$ & $91(23)$ & $87(11)$ & 0.813 \\
\hline Stroke volume (mean SD), mL & $62(26)$ & $53(20)$ & $64(29)$ & 0.177 \\
\hline Cardiac output (mean SD), L/min & $5.3(2.2)$ & $4.5(1.0)$ & $5.5(2.7)$ & 0.150 \\
\hline PPV (mean SD), \% & $10(8)$ & $14(10)$ & $6(2)$ & 0.034 \\
\hline Central Venous Pressure (mean SD), mmHg & $10(4)$ & $9(3)$ & $10(4)$ & 0.238 \\
\hline \multicolumn{5}{|l|}{ Respiratory parameters } \\
\hline Tidal volume (mean SD), mL & $436(62)$ & $452(55)$ & $405(68)$ & 0.288 \\
\hline
\end{tabular}




\begin{tabular}{|lllll|}
\hline & $\begin{array}{l}\text { Overall } \\
\text { population }\end{array}$ & Responders & $\begin{array}{l}\text { Non- } \\
\text { Responders }\end{array}$ & $\begin{array}{l}\text { p } \\
\text { value }\end{array}$ \\
\hline Respiratory Rate (mean SD), RR/min & $20(5)$ & $18(4)$ & $22(6)$ & 0.218 \\
\hline $\begin{array}{l}\text { Positive end expiratory pressure (mean SD), } \\
\text { cmH2O }\end{array}$ & $10(3)$ & $9(2)$ & $10(3)$ & 0.671 \\
\hline Plateau pressure (mean SD), cmH20 & $22(6)$ & $19(3)$ & $23(8)$ & 0.099 \\
\hline $\begin{array}{l}\text { Static pulmonary compliance (mean SD), } \\
\text { mL/cmH2O }\end{array}$ & $42(15)$ & $48(11)$ & $37(15)$ & 0.065 \\
\hline P/F (mean SD) & $236(97)$ & $224(104)$ & $242(97)$ & 0.679 \\
\hline Neuromuscular blockade, $n$ & 9 & 4 & 5 & \\
\hline Pharmacological parameters & & & & \\
\hline Norepinephrine (mean SD), ug/kg/min & $0.50(0.64)$ & $0.78(0.76)$ & $0.24(0.35)$ & 0.031 \\
\hline Dobutamine (mean SD), ug/kg/min & $2.06(3.37)$ & $1.44(2.96)$ & $2.67(3.81)$ & 0.394 \\
\hline Biological parameters & & & & \\
\hline Lactates (mean SD), mmol/l & $2.2(1.5)$ & $2.7(2.1)$ & $2.0(0.8)$ & 0.215 \\
\hline
\end{tabular}

\section{Prediction of Fluid Responsiveness}

SV values for baseline, maximum lung recruitment pressure, and before and after volume expansion are represented in figure 3.

Six data points were available for each parameter for STEP-UP and STEP-DOWN. Figure 4 shows individual variations in hemodynamic parameters during LRM. STEP-UP LRM induced a decrease of SAP, PP, DAP, MAP, and SV and an increase of CVP. Fluid Responders demonstrated a greater decrease of SAP, PP, DAP, MAP, and SV as well as a greater increase of CVP compared to Non-Responders.

Evaluation of all hemodynamic variables suggested the use of a linear regression model, especially during STEP-UP LRM. Slope calculations are shown in Table 2. Slopes are reported as aSAP, aDAP and aMAP for systolic, diastolic and mean arterial pressure, aPP for pulse pressure, aCVP for central venous pressure and aSV for stroke volume.

Slope calculations showed greater absolute values for the Responder compared to the Non-Responder group for STEP-UP and STEP-DOWN LRM (Table 2). The ability of aSAP, aPP, aDAP, aMAP, aSV and aCVP to predict fluid responsiveness and the results of AUC analysis are shown in Table 3. The best predictive variables for fluid responsiveness during LRM were aPP and aCVP during STEP-UP, with Youden indices of 0.888 and 0.777 respectively. 
aPP during STEP-UP was strongly predictive of fluid responsiveness with an AUC of $0.926(95 \% \mathrm{Cl}, 0.78$ to 1.00 ), and a sensitivity and a specificity of $100 \%$ and $89 \%$ respectively. Cut-off value was $-42.8^{\circ}$. Inconclusive values ranged from $-42.8^{\circ}$ to $-52.1^{\circ}$ using the grey zone approach ( $35 \%$ of the patients).

aCVP during STEP-UP was also strongly predictive of fluid responsiveness with an AUC of $0.901(95 \% \mathrm{Cl}$, 0.76 to 1.00 ) and a sensitivity and a specificity of $78 \%$ and $100 \%$ respectively. The cut-off value was $20.1^{\circ}$. Inconclusive values ranged from $13.8^{\circ}$ to $20.1^{\circ}$ using the grey zone approach ( $44 \%$ of the patients).

By combining sensitivity of aPP and specificity of aCVP if both measures are available, taking for each angle the specificity value of aCVP and sensitivity value of aPP, fluid responsiveness prediction can be obtained with $100 \%$ sensitivity and $100 \%$ specificity during STEP-UP LRM (AUC=0.96; $95 \% \mathrm{Cl}, 0.90$ to 1.00 ). One patient (5.5\%) showed inconclusive values using the grey zone approach (Figure 4). Index combination has already been proposed to optimize the sensitivity and specificity of a parameter (23).

Absolute variations for SAP, PP, MAP, DAP, SV and CVP between baseline (PEEP $=5 \mathrm{mmHg}$, Inspiratory pressure $=20 \mathrm{mmHg}$ ) and maximum pressure level $(P E E P=30 \mathrm{mmHg}$, Inspiratory pressure $=45 \mathrm{mmHg}$ ) are reported as $\triangle \mathrm{SAP}, \triangle \mathrm{PP}, \triangle \mathrm{MAP}, \triangle \mathrm{DAP}, \triangle \mathrm{SV}$ and $\triangle \mathrm{CVP}$. Their ability to predict fluid responsiveness is shown in table 4.

PPV at baseline was available for 11 patients. A PPV of more than $12 \%$ before LRM predicted responders with an AUC of $0.711(95 \% \mathrm{Cl}, 0.42$ to 1.00$)$ and a sensitivity and a specificity of $63 \%$ and $100 \%$ respectively. Inconclusive values ranged from $4-10 \%$ using the grey zone approach ( $45 \%$ of the patients) (Figure 5 ).

Table 2

Slopes (degrees) for systolic arterial pressure (aSAP), pulse pressure (aPP), diastolic arterial pressure $(\mathrm{aDAP})$, mean arterial pressure (aMAP), stroke volume (aSV) and central venous pressure (aCVP) for STEPUP and STEP-DOWN during lung recruitment maneuver

\begin{tabular}{|c|c|c|c|c|c|c|}
\hline & STEP-UP & & & STEP-DOWN & & \\
\hline & Responders & Non-Responders & $p$ value & Responders & Non-Responders & $p$ value \\
\hline & $N=9$ & $N=9$ & & $N=9$ & $N=9$ & \\
\hline a SAP (SD) & $\begin{array}{l}-59.4^{\circ} \\
(5.79)\end{array}$ & $-37.5^{\circ}(13.1)$ & 0.022 & $57.1^{\circ}(8.25)$ & $40.8^{\circ}(12.6)$ & 0.034 \\
\hline a PP (SD) & $\begin{array}{l}-51.9^{\circ} \\
(7.24)\end{array}$ & $-31.6^{\circ}(10.7)$ & 0.014 & $48.8^{\circ}(8.26)$ & $31.8^{\circ}(10.6)$ & 0.010 \\
\hline a DAP (SD) & $\begin{array}{l}-23.6^{\circ} \\
(8.77)\end{array}$ & $-11.4^{\circ}(10.0)$ & 0.126 & $24.3^{\circ}(9.44)$ & $18.5^{\circ}(10.1)$ & 0.528 \\
\hline $\begin{array}{l}\text { a MAP } \\
\text { (SD) }\end{array}$ & $\begin{array}{l}-40.3^{\circ} \\
(8.50)\end{array}$ & $-24.2^{\circ}(11.9)$ & 0.089 & $39.6^{\circ}(9.51)$ & $30.1^{\circ}(11.5)$ & 0.228 \\
\hline a SV (SD) & $\begin{array}{l}-43.5^{\circ} \\
(9.65)\end{array}$ & $-27.4^{\circ}(8.64)$ & 0.038 & $25.9^{\circ}(20.1)$ & $29.0^{\circ}(9.60)$ & 0.837 \\
\hline a CVP (SD) & $19.8^{\circ}(2.66)$ & $13.1^{\circ}(3.03)$ & 0.006 & $-18.2^{\circ}(2.05)$ & $-13.4^{\circ}(2.60)$ & 0.003 \\
\hline
\end{tabular}


Page 10/23 
Table 3

Diagnostic performance of slopes for systolic arterial pressure (aSAP), pulse pressure (aPP), diastolic arterial pressure (aDAP), mean arterial pressure (aMAP), stroke volume (aSV), central venous pressure $(\mathrm{aCVP})$ and relative variations from baseline of systolic arterial pressure $(\triangle \mathrm{SAP})$, pulse pressure $(\triangle \mathrm{PP})$, diastolic arterial pressure $(\triangle D A P)$, mean arterial pressure $(\triangle M A P)$, stroke volume $(\Delta S V)$ and central venous pressure $(\triangle C V P)$ between baseline $(P E E P=5$, Inspiratory Pressure $=20 \mathrm{cmH} 20)$ and maximum pressure level $(P E E P=30$, Inspiratory Pressure $=45 \mathrm{cmH} 20)$ to predict fluid responsiveness during STEP-UP and STEPDOWN lung recruitment maneuver

\begin{tabular}{|c|c|c|c|c|c|c|}
\hline STEP-UP & aSAP & aPP & aDAP & aMAP & asv & aCVP \\
\hline $\begin{array}{l}\text { Cut-off } \\
\text { value } \\
\text { (degrees) }\end{array}$ & $-47.8^{\circ}$ & $-42.8^{\circ}$ & $-10.1^{\circ}$ & $-20.1^{\circ}$ & $-34.2^{\circ}$ & $20.1^{\circ}$ \\
\hline ROC AUC & 0.864 & 0.926 & 0.765 & 0.777 & 0.854 & 0.901 \\
\hline Sensitivity & 1 & 1 & 1 & 1 & 0.875 & 0.777 \\
\hline Specificity & 0.666 & 0.888 & 0.666 & 0.666 & 0.777 & 1 \\
\hline $\begin{array}{l}\text { Positive } \\
\text { predictive } \\
\text { value }\end{array}$ & 0.75 & 0.9 & 0.75 & 0.75 & 0.777 & 1 \\
\hline $\begin{array}{l}\text { Negative } \\
\text { predictive } \\
\text { value }\end{array}$ & 1 & 1 & 1 & 1 & 0.875 & 0.818 \\
\hline $\begin{array}{l}\text { Youden } \\
\text { index }\end{array}$ & 0.666 & 0.888 & 0.666 & 0.666 & 0.653 & 0.777 \\
\hline \multirow{2}{*}{$\begin{array}{l}\text { Grey zone } \\
\text { (degrees) }\end{array}$} & {$\left[47.8^{\circ}-59.6^{\circ}\right]$} & {$\left[42.8^{\circ}-52.1^{\circ}\right]$} & {$\left[10.1^{\circ}-34.2^{\circ}\right]$} & {$\left[20.1^{\circ}-42.8^{\circ}\right]$} & {$\left[24.3^{\circ}-46.1^{\circ}\right]$} & {$\left[13.8^{\circ}-20.1^{\circ}\right]$} \\
\hline & $\Delta \mathrm{SAP}$ & $\Delta \mathrm{PP}$ & $\triangle \mathrm{DAP}$ & $\Delta \mathrm{MAP}$ & $\Delta S V$ & $\Delta \mathrm{CVP}$ \\
\hline $\begin{array}{l}\text { Cut-off } \\
\text { value } \\
\text { (mmHg) }\end{array}$ & 23 & 21 & 4 & 9 & 16 & 8 \\
\hline ROC AUC & 0.901 & 0.920 & 0.777 & 0.790 & 0.753 & 0.883 \\
\hline Sensitivity & 1 & 1 & 1 & 1 & 0.777 & 0.777 \\
\hline Specificity & 0.666 & 0.777 & 0.666 & 0.666 & 0.777 & 0.888 \\
\hline $\begin{array}{l}\text { Positive } \\
\text { predictive } \\
\text { value }\end{array}$ & 0.75 & 0.818 & 0.75 & 0.75 & 0.777 & 0.875 \\
\hline $\begin{array}{l}\text { Negative } \\
\text { predictive } \\
\text { value }\end{array}$ & 1 & 1 & 1 & 1 & 0.777 & 0.8 \\
\hline $\begin{array}{l}\text { Youden } \\
\text { index }\end{array}$ & 0.666 & 0.777 & 0.666 & 0.666 & 0.555 & 0.666 \\
\hline $\begin{array}{l}\text { Grey zone } \\
(\mathrm{mmHg})\end{array}$ & [23-39] & [21-28] & [4-14] & [9-23] & [0-25] & [5-9] \\
\hline
\end{tabular}




\begin{tabular}{|c|c|c|c|c|c|c|}
\hline STEP-UP & aSAP & aPP & aDAP & aMAP & aSV & aCVP \\
\hline $\begin{array}{l}\text { STEP- } \\
\text { DOWN }\end{array}$ & aSAP & aPP & aDAP & aMAP & aSV & aCVP \\
\hline $\begin{array}{l}\text { Cut-off } \\
\text { value } \\
\text { (degrees) }\end{array}$ & $55.4^{\circ}$ & $47.8^{\circ}$ & $20.4^{\circ}$ & $34.7^{\circ}$ & $43.2^{\circ}$ & $-14.1^{\circ}$ \\
\hline ROC AUC & 0.777 & 0.815 & 0.666 & 0.685 & 0.666 & 0.877 \\
\hline Sensitivity & 0.777 & 0.666 & 0.777 & 0.777 & 0.375 & 0.888 \\
\hline Specificity & 0.777 & 0.888 & 0.666 & 0.666 & 1 & 0.777 \\
\hline $\begin{array}{l}\text { Positive } \\
\text { predictive } \\
\text { value }\end{array}$ & 0.777 & 0.857 & 0.7 & 0.7 & 1 & 0.8 \\
\hline $\begin{array}{l}\text { Negative } \\
\text { predictive } \\
\text { value }\end{array}$ & 0.777 & 0.727 & 0.75 & 0.75 & 0.643 & 0.875 \\
\hline $\begin{array}{l}\text { Youden } \\
\text { index }\end{array}$ & 0.555 & 0.555 & 0.444 & 0.444 & 0.375 & 0.666 \\
\hline \multirow{2}{*}{$\begin{array}{l}\text { Grey zone } \\
\text { (degrees) }\end{array}$} & {$\left[38.7^{\circ}-67.8^{\circ}\right]$} & {$\left[32.4^{\circ}-52.3^{\circ}\right]$} & {$\left[3.9^{\circ}-36.0^{\circ}\right]$} & {$\left[9.76^{\circ}-50.6^{\circ}\right]$} & {$\left[13.2^{\circ}-43.2^{\circ}\right]$} & {$\left[13.8^{\circ}-20.1^{\circ}\right]$} \\
\hline & $\Delta \mathrm{SAP}$ & $\Delta \mathrm{PP}$ & $\Delta \mathrm{DAP}$ & $\Delta \mathrm{MAP}$ & $\Delta S V$ & $\Delta \mathrm{CVP}$ \\
\hline $\begin{array}{l}\text { Cut-off } \\
\text { value } \\
\text { (mmHg) }\end{array}$ & 32 & 21 & 8 & 17 & 15 & 5 \\
\hline ROC AUC & 0.809 & 0.852 & 0.741 & 0.735 & 0.642 & 0.888 \\
\hline Sensitivity & 0.777 & 0.777 & 0.777 & 0.777 & 0.666 & 1 \\
\hline Specificity & 0.888 & 0.888 & 0.777 & 0.777 & 0.666 & 0.666 \\
\hline $\begin{array}{l}\text { Positive } \\
\text { predictive } \\
\text { value }\end{array}$ & 0.875 & 0.875 & 0.777 & 0.777 & 0.666 & 0.75 \\
\hline $\begin{array}{l}\text { Negative } \\
\text { predictive } \\
\text { value }\end{array}$ & 0.8 & 0.8 & 0.777 & 0.777 & 0.666 & 1 \\
\hline $\begin{array}{l}\text { Youden } \\
\text { index }\end{array}$ & 0.666 & 0.666 & 0.555 & 0.555 & 0.333 & 0.666 \\
\hline $\begin{array}{l}\text { Grey zone } \\
(\mathrm{mmHg})\end{array}$ & [11-46] & [13-30] & [0-16] & [4-29] & [0-25] & [5-8] \\
\hline
\end{tabular}

\section{Discussion}


In our study, results show that aCVP and aPP changes induced by a progressive lung pressure (STEP-PEEP) recruitment maneuver are the best hemodynamic parameters for the prediction of fluid responsiveness in mechanically ventilated patients in ICU, with or without neuromuscular blockade.

STEP-PEEP LRM offered the ability to evaluate the effects of LRM using a different approach from usual bilevel sustained insufflation LRM. Six data points were available for STEP-UP and STEP-DOWN for all parameters, following a linear evolution especially during STEP-UP, allowing a linear regression analysis. Absolute variations of PP and CVP between PEEP $=5 \mathrm{cmH} 20$ and PEEP $=30 \mathrm{cmH} 20$ during STEP-UP provide interesting results with respect to discrimination of fluid responders and non-responders. PPV was significantly different between Responders and Non-Responders at baseline but showed inferior statistical values compared to slope analysis.

During LRM, a transient increase in intra-pulmonary pressure is transmitted to the adjacent intra-thoracic compartments. This increase in intrathoracic pressure most impacts the right ventricle, however no patients in our study had right ventricular cardiac dysfunction as they were excluded. Preload and afterload of the right ventricle are also affected by high intra-thoracic pressure. High intra-thoracic pressure increases the right ventricular afterload and ejectional impedance. (25) These effects are particularly significant when preload is low. $(23,26)$ All patients in our study displayed a significant decrease in SV and systemic arterial pressure, and an increase in CVP during LRM. Hemodynamic changes were more important in the Responders group.

Cardio-pulmonary interactions are widely studied and used in clinical practice, especially with PPV analysis and more recently with LRM hemodynamic response analysis $(12,13,14)$ or end expiratory occlusion test (27). Pressure transmission from lung and pleural space to the heart and mediastinum can be affected by many physiological and physio-pathological conditions. Compliance loss in acute respiratory distress syndrome for patients in intensive care units or catecholamine use can also lead to changes in cardiopulmonary interactions. Therefore, it is important for clinicians not to oversimplify this physiology and to take into account the characteristics of the underlying pathologies before making any decision based on the scientific literature.

PP slope calculation (aPP) after linear regression during STEP-PEEP showed excellent sensitivity. This can be explained by the fact that if a transient increase in intra-thoracic pressure does not impact systemic arterial pressure, the right ventricular preload is likely to be sufficient to avoid collapse. aPP variation below cut-off value during STEP-UP could therefore exclude fluid responsiveness with a 100\% sensitivity in our study.

CVP slope calculation (aCVP) during STEP-LRM showed excellent specificity. This can be explained by the fact that if a transient increase in intra-thoracic pressure induces a significant variation in CVP, the right ventricular preload is likely to be low and easily affected external pressure. aCVP above cut-off value during STEP-UP can confirm fluid responsiveness with $100 \%$ specificity in our study.

Clinicians may therefore benefit from the sensitivity of aPP using only an arterial catheter, or the specificity of aCVP using a central venous catheter for fluid responsiveness prediction. For patients equipped with 
both, combination of aPP and aCVP during STEP-UP allows clinicians to predict fluid responsiveness with $100 \%$ sensitivity and specificity.

This study has several limitations. This pilot study was monocentric and 18 only patients have been studied. Statistical results are highly significant but further larger studies are needed to confirm our preliminary results. Next, SV during LRM was calculated with pulse contour analysis. Surprisingly, the SV results for absolute variation and angle analysis were inferior to results for SAP, PP and CVP. SV calculated from pulse contour analysis may have been imprecise due to increasing levels of pressure and variation of systemic vascular resistances from baseline initial transcardiopulmonary thermodilution. Of note, thermodilution is the method of choice for cardiac output measurement and was used before LRM, and after fluid challenge.

Baseline norepinephrine differed between the R and NR groups. Differences of norepinephrine dosage may have had an effect on the stressed and unstressed fluid volume, potentially leading to bias in interpretation of CVP for evaluation of fluid responsiveness.

All included patients required fluid expansion as determined by the clinician in charge, based on vasopressor requirements, echocardiographic evidence or clinical judgement. Half of the patients included were found to have received suboptimal fluid management, illustrating the need for a specific tool for evaluation of fluid responsiveness.

Calculation of the slope may be challenging at bedside but a calculation of the $a$ angle according to the successive values of CVP and PP can easily be automated on a computer or smartphone.

All patients were not consecutive patients, introducing a potential selection bias. Before inclusion, all patients required a fluid expansion as determined by the clinician in charge based on vasopressor requirements, echocardiographic evidence or clinical judgement. Inclusions were conducted exactly the same way for all patients, Responder or Non-Responder status being determined offline only after fluid expansion. The fact that half of the included patients were Non-Responders shows that our current tools are not accurate enough to discriminate Responders and Non-Responders.

The use and format of LRM can be debated. High intrathoracic pressures can have harmful effects on patients without lung disease. The STEP-PEEP lung recruitment maneuver was chosen from the literature as it showed lesser increase in transpulmonary pressure for a longer period of time and improved lung aeration as effectively as sustained inflation does, with less risk of hemodynamic compromise and hyperinflation. $(16,17)$

\section{Conclusion}

In mechanically ventilated patients, a progressive STEP-PEEP lung recruitment maneuver could predict fluid responsiveness using the slope analysis of pulse pressure (aPP) and central venous pressure (aCVP) evolutions. aPP variation below cut-off value during STEP-UP can exclude fluid responsiveness. aCVP above cut-off value during STEP-UP can affirm fluid responsiveness. In this pilot study, the association of

Page 14/23 
aPP and aCVP during STEP-UP recruitment maneuver provides a high sensitivity and high specificity and seems to offer a very promising method for fluid responsiveness prediction without the use and cost of a cardiac output measurement device.

\section{Abbreviations}

LRM: Lung Recruitment Maneuver

CVP: Central Venous Pressure

SV: Stroke Volume

PP: Pulse Pressure

PPV: Pulse Pressure Variation

SAP: Systolic Arterial Pressure

MAP: Mean Arterial Pressure

DAP: Diastolic Arterial Pressure

ICU: Intensive Care Unit

TV: Tidal Volume

CO: Cardiac Output

R: Responders (to volume expansion)

NR: Non Resonders (to volume expansion)

aSAP: angle calculation between $\mathrm{X}$ axis and SAP regression interpolation line aMAP: angle calculation between $\mathrm{X}$ axis and MAP regression interpolation line aDAP: angle calculation between $\mathrm{X}$ axis and DAP regression interpolation line aPP: angle calculation between $\mathrm{X}$ axis and PP regression interpolation line aCVP: angle calculation between $\mathrm{X}$ axis and CVP regression interpolation line aSV: angle calculation between $\mathrm{X}$ axis and SV regression interpolation line SD: Standard deviation

AUC: Area Under Curve 
$\triangle$ SAP: Absolute variation for SAP

$\Delta \mathrm{PP}:$ Absolute variation for $\mathrm{PP}$

$\triangle \mathrm{DAP}:$ Absolute variation for DAP

$\triangle \mathrm{MAP}:$ Absolute variation for MAP

$\triangle$ CVP: Absolute variation for CVP

$\Delta S V$ : Absolute variation for SV

\section{Declarations}

\section{Ethical Approval and Consent to participate}

The study protocol was approved by the hospital's ethics committee (Ethics Committee, Department of Anesthesiology, Saint-Etienne University Central Hospital, institutional Review Board IORG0007394, Protocol number IRBN902018/CHUSTE).

\section{Consent for publication}

Not applicable

\section{Availability of data and materials}

The datasets used and/or analyzed during the current study are available from the corresponding author on reasonable request.

\section{Competing interests}

The authors declare that they have no competing interests

\section{Funding}

Department of Anesthesiology, Saint-Etienne University Central Hospital

\section{Authors' contributions}

SV designed, included and made statistic calculations for this study.

JBB designed and included patients.

OD reviewed the article and made significant corrections.

CF included patients.

DR reviewed and corrected english syntax. 
BT reviewed the article and made significant corrections.

LG designed, included and made statistical calculations.

JM made this work possible in his intensive care unit and made significant corrections to the manuscript.

All authors read and approved the final manuscript

\section{Acknowledgements}

Not applicable

\section{ETHICAL APPROVAL AND CONSENT TO PARTICIPATE}

All methods were carried out in accordance with relevant guidelines and regulations. The study protocol was approved by the hospital's ethics committee (Ethics Committee, Department of Anesthesiology, SaintEtienne University Central Hospital, institutional Review Board IORG0007394, Protocol number IRBN902018/CHUSTE). Written informed consent was obtained for all study patients or relatives if indicated. Oral consent was obtained and reported in the medical record.

Trial registration: NCT04304521, IRBN902018/CHUSTE

Registered 11 March 2020, Fluid responsiveness predicted by a stepwise PEEP elevation recruitment maneuver in mechanically ventilated patients (STEP-PEEP)

https://www.clinicaltrials.gov/ct2/show/NCT04304521?term $=$ NCT04304521\&cntry=FR\&draw=2\&rank=1

\section{FUNDING}

No funding was received for this study

\section{References}

1. Giglio M, Dalfino L, Puntillo F, Brienza N. Hemodynamic goal-directed therapy and postoperative kidney injury: an updated meta-analysis with trial sequential analysis. Crit Care. 2019;23(1):232. doi:10.1186/s13054-019-2516-4

2. Marik PE, Baram M, Vahid B. Does central venous pressure predict fluid responsiveness? A systematic review of the literature and the tale of seven mares. Chest. 2008;134(1):172-178. doi:10.1378/chest.072331

3. Deng Q-W, Tan W-C, Zhao B-C, Wen S-H, Shen J-T, Xu M. Is goal-directed fluid therapy based on dynamic variables alone sufficient to improve clinical outcomes among patients undergoing surgery? A metaanalysis. Crit Care. 2018;22(1):298. doi:10.1186/s13054-018-2251-2

4. Michard F, Chemla D, Teboul J-L. Applicability of pulse pressure variation: how many shades of grey? Crit Care. 2015;19:144. doi:10.1186/s13054-015-0869-x 
5. Perel A, Minkovich L, Preisman S, Abiad M, Segal E, Coriat P. Assessing fluid-responsiveness by a standardized ventilatory maneuver: the respiratory systolic variation test. Anesth Analg. avr 2005; $100(4): 942-5$

6. Jozwiak M, Monnet X, Teboul J-L. Prediction of fluid responsiveness in ventilated patients. Ann Transl Med. 2018;6(18):352. doi:10.21037/atm.2018.05.03

7. Güldner A, Kiss T, Serpa Neto A, et al. Intraoperative protective mechanical ventilation for prevention of postoperative pulmonary complications: a comprehensive review of the role of tidal volume, positive end-expiratory pressure, and lung recruitment maneuvers. Anesthesiology. 2015;123(3):692-713. doi:10.1097/ALN.0000000000000754

8. Nielsen J, Østergaard M, Kjaergaard J, et al. Lung recruitment maneuver depresses central hemodynamics in patients following cardiac surgery. Intensive Care Med. 2005;31(9):1189-1194. doi:10.1007/s00134-005-2732-z

9. Luecke T, Pelosi P. Clinical review: Positive end-expiratory pressure and cardiac output. Crit Care. 2005;9(6):607-621. doi:10.1186/cc3877

10. Michard F, Chemla D, Richard $C$, et al. Clinical use of respiratory changes in arterial pulse pressure to monitor the hemodynamic effects of PEEP. Am J Respir Crit Care Med. 1999;159(3):935-939. doi:10.1164/ajrccm.159.3.9805077

11. Biais M, Nouette-Gaulain K, Quinart A, Roullet S, Revel P, Sztark F. Uncalibrated stroke volume variations are able to predict the hemodynamic effects of positive end-expiratory pressure in patients with acute lung injury or acute respiratory distress syndrome after liver transplantation. Anesthesiology. 2009;111(4):855-862. doi:10.1097/ALN.0b013e3181b27fb2

12. Biais M, Lanchon R, Sesay M, et al. Changes in Stroke Volume Induced by Lung Recruitment Maneuver Predict Fluid Responsiveness in Mechanically Ventilated Patients in the Operating Room. Anesthesiology. 2017;126(2):260-267. doi:10.1097/ALN.0000000000001459

13. De Broca B, Garnier J, Fischer M-O, et al. Stroke volume changes induced by a recruitment maneuver predict fluid responsiveness in patients with protective ventilation in the operating theater. Medicine (Baltimore). 2016;95(28):e4259. doi:10.1097/MD.0000000000004259

14. Wilkman E, Kuitunen A, Pettilä V, Varpula M. Fluid responsiveness predicted by elevation of PEEP in patients with septic shock. Acta Anaesthesiol Scand. 2014;58(1):27-35. doi:10.1111/aas. 12229

15. Joosten A, Desebbe 0 , Suehiro $K$, et al. Accuracy and precision of non-invasive cardiac output monitoring devices in perioperative medicine: a systematic review and meta-analysist. Br J Anaesth. 2017;118(3):298-310. doi:10.1093/bja/aew461

16. Constantin J-M, Godet T, Jabaudon M, Bazin J-E, Futier E. Recruitment maneuvers in acute respiratory distress syndrome. Ann Trans/ Med. 2017;5(14):290. doi:10.21037/atm.2017.07.09

17. Santos RS, Silva PL, Pelosi P, Rocco PR. Recruitment maneuvers in acute respiratory distress syndrome: The safe way is the best way. World J Crit Care Med. 2015;4(4):278-286. doi:10.5492/wjccm.v4.i4.278

18. Messina A, Colombo D, Barra FL, et al. Sigh maneuver to enhance assessment of fluid responsiveness during pressure support ventilation. Crit Care. 2019;23(1):31. doi:10.1186/s13054-018-2294-4 
19. Constantin J-M, Jaber S, Futier E, et al. Respiratory effects of different recruitment maneuvers in acute respiratory distress syndrome. Crit Care. 2008;12(2):R50. doi:10.1186/cc6869

20. Guinot $\mathrm{P}-\mathrm{G}$, Urbina $\mathrm{B}$, de Broca $\mathrm{B}$, Bernard E, Dupont $\mathrm{H}$, Lorne E. Predictability of the respiratory variation of stroke volume varies according to the definition of fluid responsiveness. Br J Anaesth. 2014;112(3):580-581. doi:10.1093/bja/aeu031

21. Cannesson M, Le Manach Y, Hofer CK, et al. Assessing the diagnostic accuracy of pulse pressure variations for the prediction of fluid responsiveness: a "gray zone" approach. Anesthesiology. 2011;115(2):231-241. doi:10.1097/ALN.0b013e318225b80a

22. DeLong ER, DeLong DM, Clarke-Pearson DL. Comparing the areas under two or more correlated receiver operating characteristic curves: a nonparametric approach. Biometrics. 1988;44(3):837-845.

23. Keller G, Sinavsky K, Desebbe O, Lehot JJ. Combination of continuous pulse pressure variation monitoring and cardiac filling pressure to predict fluid responsiveness. J Clin Monit Comput. 2012 Dec;26(6):401-5. doi: 10.1007/s10877-012-9365-x. Epub 2012 May 3. PMID: 22552875.

24. Fougères E, Teboul J-L, Richard C, Osman D, Chemla D, Monnet $X$. Hemodynamic impact of a positive end-expiratory pressure setting in acute respiratory distress syndrome: importance of the volume status. Crit Care Med. 2010;38(3):802-807. doi:10.1097/CCM.0b013e3181c587fd

25. Jardin F, Farcot JC, Boisante L, Curien N, Margairaz A, Bourdarias JP. Influence of positive endexpiratory pressure on left ventricular performance. N Engl J Med. 1981;304(7):387-392. doi:10.1056/NEJM198102123040703

26. Nielsen J, Østergaard M, Kjaergaard J, et al. Lung recruitment maneuver depresses central hemodynamics in patients following cardiac surgery. Intensive Care Med. 2005;31(9):1189-1194. doi:10.1007/s00134-005-2732-z

27. Gavelli F, Shi R, Teboul J-L, Azzolina D, Monnet X. The end-expiratory occlusion test for detecting preload responsiveness: a systematic review and meta-analysis. Ann Intensive Care. 2020;10(1):65. doi:10.1186/s13613-020-00682-8

\section{Table}

Table 4 is not available with this version.

\section{Figures}




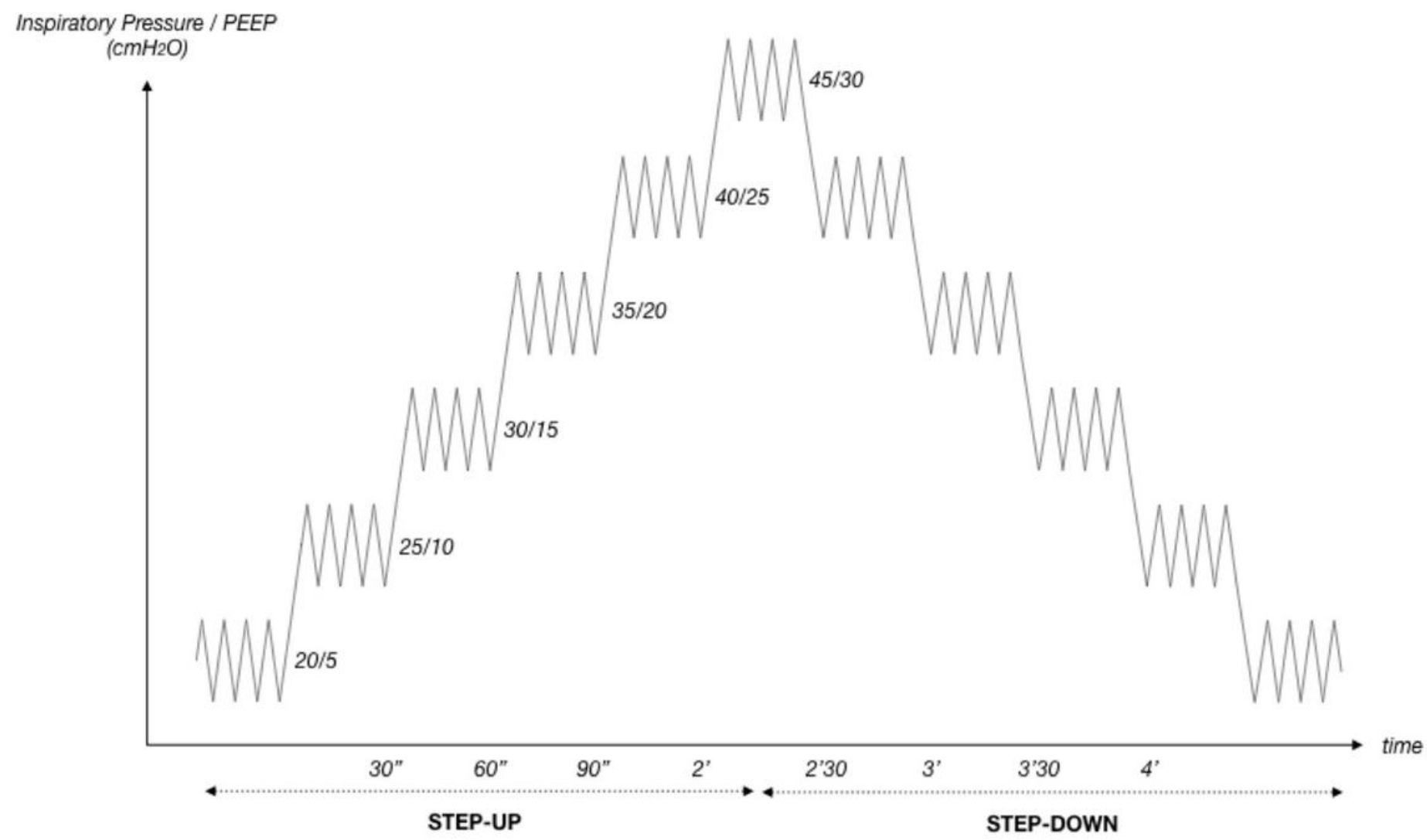

Figure 1

Schematic representation of STEP-PEEP lung recruitment maneuver 


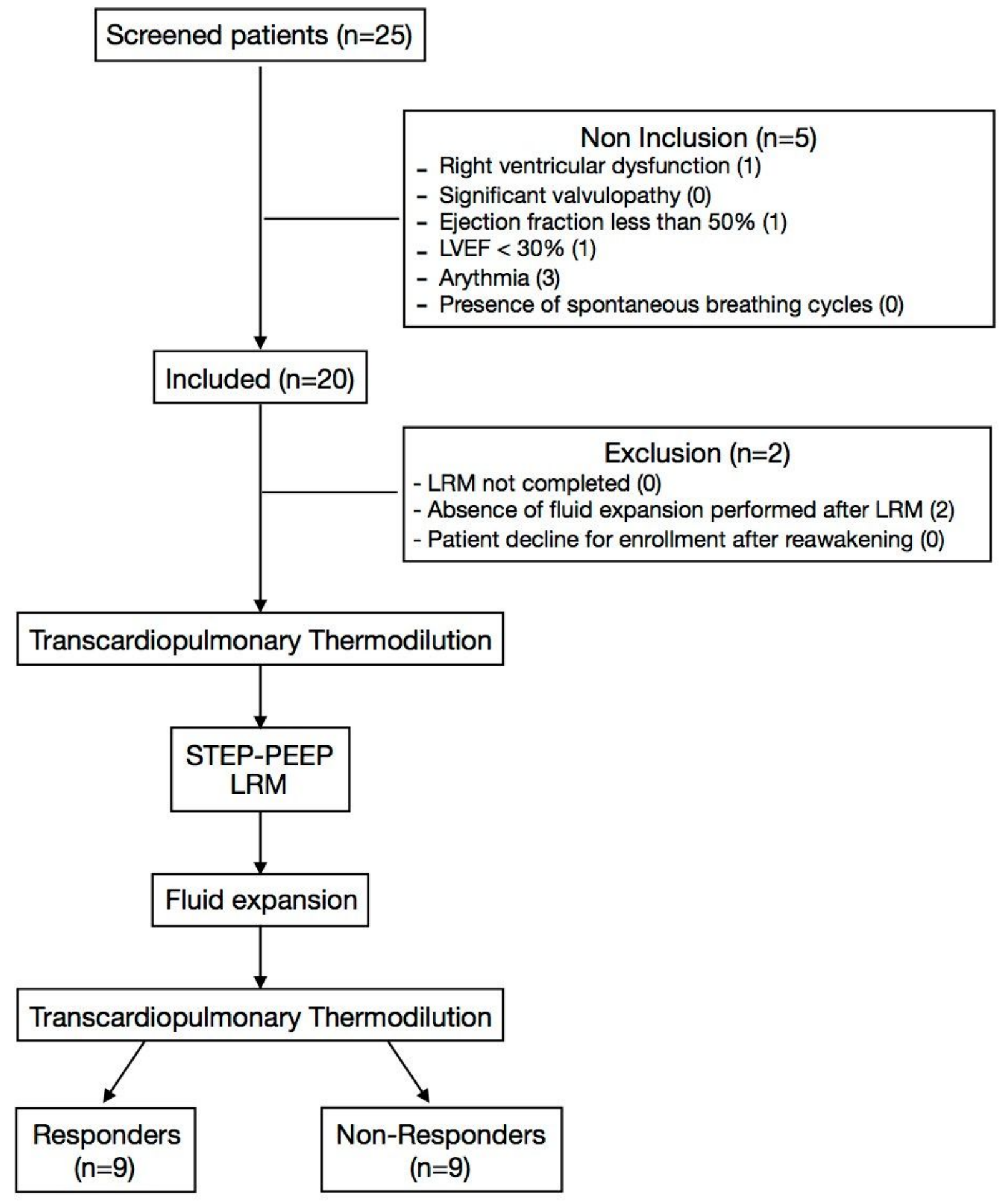

Figure 2

Study flow chart 


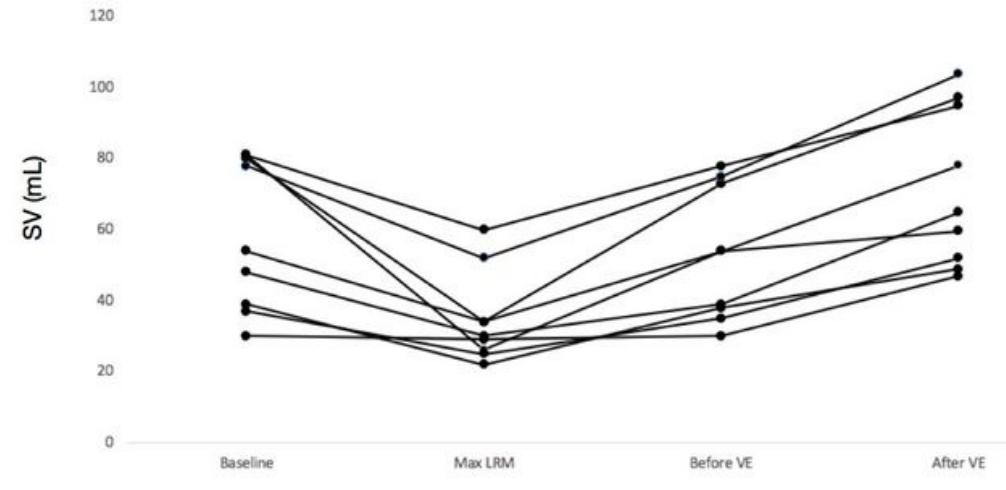

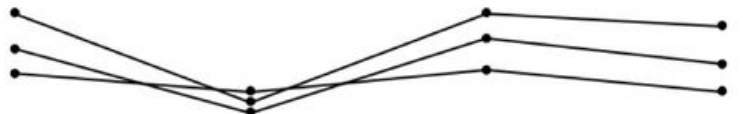

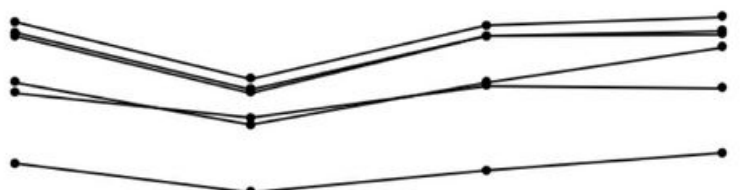

Baseline

Max LRM

\section{Figure 3}

Representation of SV $(\mathrm{mL})$ values for each step: baseline, maximum pressure level during LRM, before VE and after VE
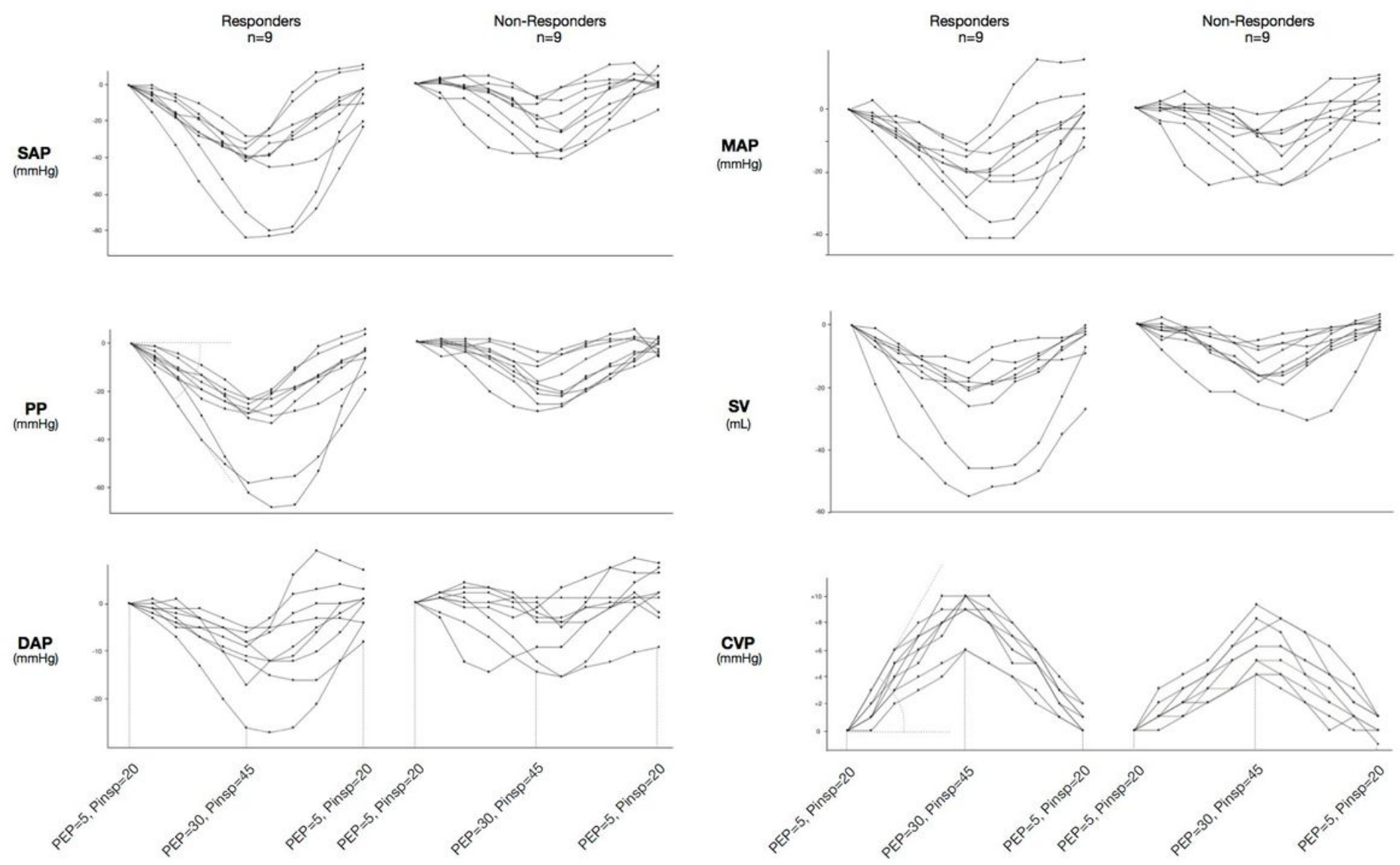

\section{Figure 4}

Line graphs showing the relationship between PEEP and change in pressure through the stepwise lung recruitment maneuver and example of alpha angle calculation between the $\mathrm{X}$ axis and the regression 
interpolation line.
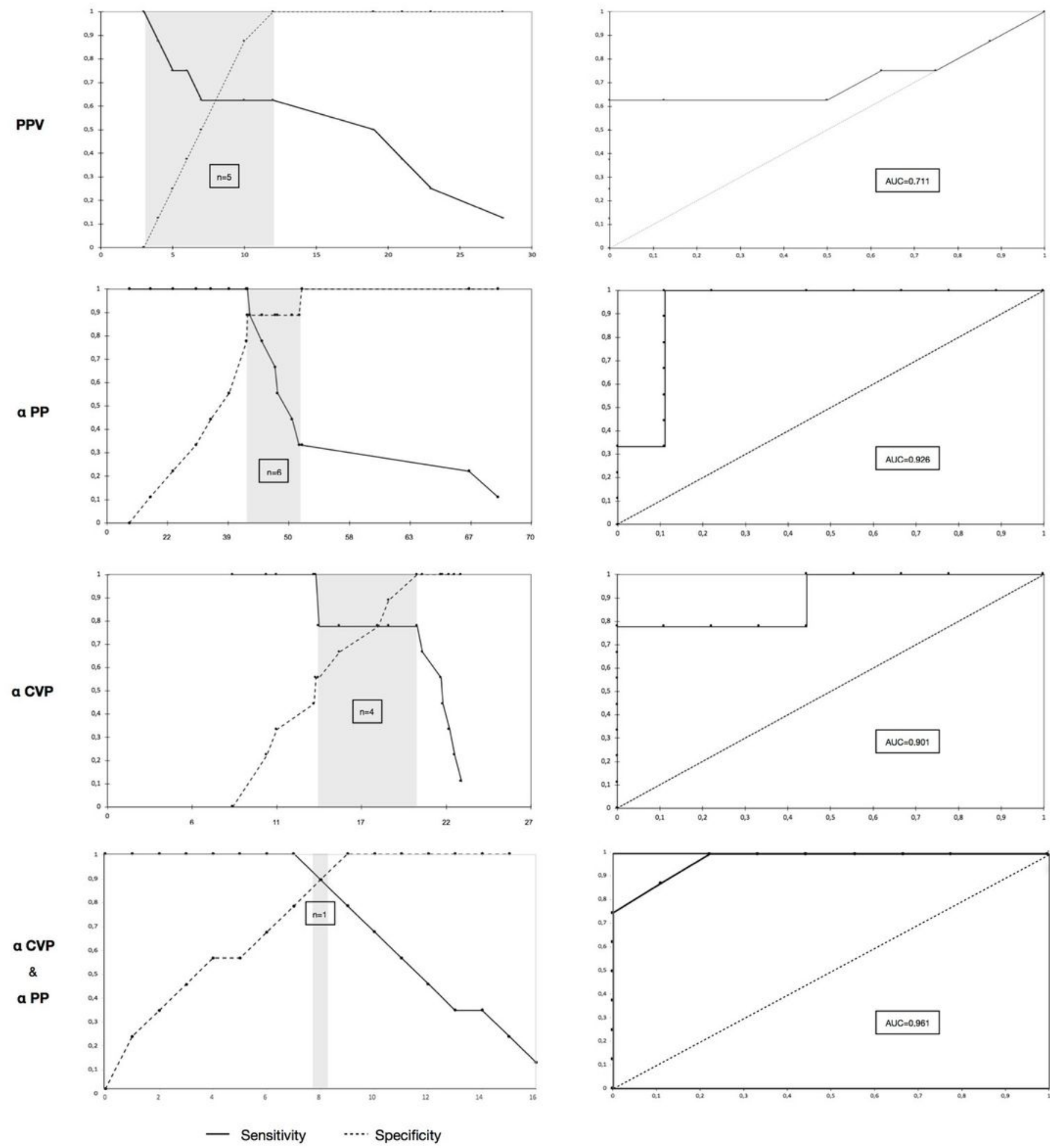

\section{Figure 5}

Sensitivity, specificity, grey zone and Receiver Operating Curves generated of pulse pressure variation (PPV) at baseline and slopes for changes in pulse pressure (aPP), central venous pressure (aCVP) and combination of aPP sensitivity \& aCVP specificity during STEP-UP lung recruitment maneuver, with a view to discriminating between fluid expansion Responders and Non-Responders 